\title{
Intermolecular, Markovnikov Hydroamination of Vinylarenes with Alkylamines
}

\author{
Supporting Information
}

Masaru Utsunomiya and John F Hartwig*

Yale University, Department of Chemistry, P.O. Box 208107, New Haven, CT 06520-8107 
General comments. All reactions were performed under a nitrogen atmosphere using standard Schlenk and drybox techniques. Toluene, diethyl ether, and pentane were distilled from sodium and benzophenone under nitrogen. Vinylarenes, amines, ligands, triflic acid, and nonafluorobutane sulfonic acid were purchased from commercial sources and were used without further purification. $\operatorname{Pd}(\mathrm{DPPF})(\mathrm{OTf})_{2}{ }^{1}$ and $\mathrm{Pd}(\mathrm{DPPF})_{2}{ }^{2}$ were synthesized by literature procedures. NMR spectra were recorded on Bruker DPX 400 and $500 \mathrm{MHz}$ instruments. Elemental analyses were performed by Robertson Microlit Laboratories, Madison, NJ.

General Procedure for the Hydroamination of Vinylarenes with Alkyl Amines (Table 2). In a drybox, $\mathrm{Pd}\left(\mathrm{O}_{2} \mathrm{CCF}_{3}\right)_{2}$ and 1,1'-Bis(diphenylphosphino)ferrocene were suspended in $0.25 \mathrm{~mL}$ of dioxane in a screw-capped vial. Vinylarenes $(2.00 \mathrm{mmol})$ and amines $(1.00 \mathrm{mmol})$ were placed into a second small vial and dissolved in $0.25 \mathrm{~mL}$ of 1,4-dioxane. The vials were sealed with a cap containing a PTFE septum and removed from the drybox. Triflic acid $(0.20$ mmol) was added to the catalyst suspension. The solution of vinylarenes and amines was then added to the catalyst suspension. The reaction mixture was stirred at $120{ }^{\circ} \mathrm{C}$ for $24 \mathrm{~h}$. After heating, the reaction mixture was directly adsorbed onto silica gel and purified by flash column chromatography.

$N$-(1-Phenethyl)morpholine (Table 2, Entry 1). ${ }^{3}$ The general procedure was followed with styrene $(229 \square 1,2.00 \mathrm{mmol})$ and morpholine $(87.2 \mathrm{mg}, 1.00 \mathrm{mmol})$. The reaction mixture was purified by flash column chromatography (10\% EtOAc in hexane) to give $144 \mathrm{mg}(75 \%)$ of the hydroamination product: ${ }^{1} \mathrm{H}$ NMR (400 $\left.\mathrm{MHz} \mathrm{CDCl}_{3}, \mathrm{TMS}\right) \square 1.45$ (d, $\left.J=6.7 \mathrm{~Hz}, 3 \mathrm{H}\right), 2.35$ $(\mathrm{m}, 2 \mathrm{H}), 2.47(\mathrm{~m}, 2 \mathrm{H}), 3.29(\mathrm{q}, J=6.7 \mathrm{~Hz}, 1 \mathrm{H}), 3.68(\mathrm{t}, \mathrm{J}=4.9 \mathrm{~Hz}, 4 \mathrm{H}), 7.23(\mathrm{~m}, 1 \mathrm{H}), 7.31(\mathrm{~m}$, $4 \mathrm{H}) ;{ }^{13} \mathrm{C}\left\{{ }^{1} \mathrm{H}\right\} \mathrm{NMR}\left(100 \mathrm{MHz}, \mathrm{CDCl}_{3}\right) \square 19.8,51.2,65.3,67.2,126.9,127.6,128.2,143.9$.

$N$-[1-(2,4-Dimethylphenyl)ethyl]morpholine (Table 2, Entry 2). The general procedure was followed with 2,4-dimethylstyrene $(584 \square 1,4.00 \mathrm{mmol})$ and morpholine $(86.8 \mathrm{mg}$, $1.00 \mathrm{mmol}$ ). The reaction mixture was stirred at $120{ }^{\circ} \mathrm{C}$ for $48 \mathrm{~h}$. The reaction mixture was purified by flash column chromatography (5\% EtOAc in hexane) to give $111 \mathrm{mg}(51 \%)$ of the hydroamination product. ${ }^{1} \mathrm{H}$ NMR (400 MHz, $\left.\mathrm{CDCl}_{3}, \mathrm{TMS}\right) \square 1.26(\mathrm{~d}, J=6.6 \mathrm{~Hz}, 3 \mathrm{H}), 2.28$ (s, $3 \mathrm{H}), 2.32$ (s, 3H), 2.31-2.41 (m, 2H), 2.43-2.54 (m, 2H), $3.49(\mathrm{q}, J=6.6 \mathrm{~Hz}, 1 \mathrm{H}), 3.60-3.74(\mathrm{~m}$, 4H), $6.94(\mathrm{~s}, 1 \mathrm{H}), 6.99(\mathrm{~d}, J=7.9 \mathrm{~Hz}, 1 \mathrm{H}), 7.31(\mathrm{~d}, J=7.9 \mathrm{~Hz}, 1 \mathrm{H}) ;{ }^{13} \mathrm{C}\left\{{ }^{1} \mathrm{H}\right\} \mathrm{NMR}(100 \mathrm{MHz}$, $\left.\mathrm{CDCl}_{3}\right) \square 18.6,19.4,20.9,51.3,60.6,67.3,126.7,126.7,131.1$, 135.6, 135.8, 139.5; Anal. Calcd for $\mathrm{C}_{14} \mathrm{H}_{21} \mathrm{NO}$ : C, 76.67; H, 9.65; N, 6.39. Found: C, 76.59; H, 9.70; N, 6.25. 
$N$-[1-(4-Methoxyphenyl)ethyl]morpholine (Table 2, Entry 3). The general procedure was followed with 4-methoxystyrene (266 $\square 1,2.00 \mathrm{mmol})$ and morpholine $(87.5 \mathrm{mg}, 1.00$ mmol). The reaction mixture was purified by flash column chromatography (20\% EtOAc in hexane) to give $168 \mathrm{mg}(76 \%)$ of the hydroamination product. ${ }^{1} \mathrm{H} \mathrm{NMR}\left(400 \mathrm{MHz}, \mathrm{CDCl}_{3}\right.$, TMS) $\square 1.33(\mathrm{~d}, J=6.8 \mathrm{~Hz}, 3 \mathrm{H}), 2.29-2.39(\mathrm{~m}, 2 \mathrm{H}), 2.40-2.52(\mathrm{~m}, 2 \mathrm{H}), 3.26(\mathrm{q}, J=6.8 \mathrm{~Hz}, 1 \mathrm{H})$, $3.68(\mathrm{t}, J=4.7 \mathrm{~Hz}, 4 \mathrm{H}) 3.79(\mathrm{~s}, 3 \mathrm{H}), 6.78-6.88(\mathrm{~m}, 2 \mathrm{H}), 7.16-7.26(\mathrm{~m}, 2 \mathrm{H}) ;{ }^{13} \mathrm{C}\left\{{ }^{1} \mathrm{H}\right\} \mathrm{NMR}(100$ $\left.\mathrm{MHz}, \mathrm{CDCl}_{3}\right) \square 19.7,51.2,55.1,64.6,67.2,113.5,128.6$, 135.7, 158.4; Anal. Calcd for $\mathrm{C}_{13} \mathrm{H}_{19} \mathrm{NO}_{2}$ : C, 70.56; H, 8.65; N, 6.33. Found: C, 70.47; H, 8.44; N, 6.25.

$N$-[1-(2-naphthyl)ethyl]morpholine (Table 2, Entry 4). The general procedure was followed with 2-vinylnapthalene $(308 \mathrm{mg}, 2.00 \mathrm{mmol})$ and morpholine $(87.0 \mathrm{mg}, 0.99 \mathrm{mmol})$. The reaction mixture was stirred at $100{ }^{\circ} \mathrm{C}$ for $24 \mathrm{~h}$ and was then purified by flash column chromatography (10\% EtOAc in hexane) to give $190 \mathrm{mg}(79 \%)$ of the hydroamination product. ${ }^{1} \mathrm{H}$ NMR (400 MHz, $\mathrm{CDCl}_{3}$, TMS) $\square 1.41$ (d, $\left.J=6.6 \mathrm{~Hz}, 3 \mathrm{H}\right), 2.32-2.44$ (m, 2H), 2.46-2.60 (m, 2H), 3.43 (q, $J=6.6 \mathrm{~Hz}, 1 \mathrm{H}), 3.62-3.78(\mathrm{~m}, 4 \mathrm{H}), 7.39-7.54(\mathrm{~m}, 3 \mathrm{H}), 7.7(\mathrm{~s}, 1 \mathrm{H}), 7.74-7.88$ (m, $3 \mathrm{H}) ;{ }^{13} \mathrm{C}\left\{{ }^{1} \mathrm{H}\right\} \mathrm{NMR}\left(100 \mathrm{MHz}, \mathrm{CDCl}_{3}\right) \square 19.8,51.4,65.5,67.1,125.5,125.7,125.9,126.1$, 127.5, 127.6, 128.0, 132.7, 133.3, 141.6; Anal. Calcd for $\mathrm{C}_{16} \mathrm{H}_{19} \mathrm{NO}$ : C, 79.63; H, 7.94; N, 5.80. Found: C, 79.91; H, 7.77; N, 5.50.

$N$-[1-(3-Trifluoromethylphenyl)ethyl]morpholine (Table 2, Entry 5). The general procedure was followed with 3-trifluoromethylstyrene (593 $\square 1,4.00 \mathrm{mmol})$ and morpholine (87.0 $\mathrm{mg}, 0.99 \mathrm{mmol}$ ), and $0.20 \mathrm{~mL}$ of 1,4-dioxane. The reaction mixture was purified by flash column chromatography (20\% EtOAc in hexane) to give $132 \mathrm{mg}(51 \%)$ of the hydroamination product. ${ }^{1} \mathrm{H}$ NMR (400 MHz, $\mathrm{CDCl}_{3}$, TMS) $\square 1.35$ (d, $\left.J=6.6 \mathrm{~Hz}, 3 \mathrm{H}\right), 2.27-2.39$ (m, 2H), 2.42-2.55 (m, 2H), $3.37(\mathrm{q}, J=6.6 \mathrm{~Hz}, 1 \mathrm{H}), 3.62-3.77(\mathrm{~m}, 4 \mathrm{H}), 7.39-7.46(\mathrm{~m}, 1 \mathrm{H}), 7.47-7.55(\mathrm{~m}, 2 \mathrm{H}), 7.59$ (s, $1 \mathrm{H}) ;{ }^{13} \mathrm{C}\left\{{ }^{1} \mathrm{H}\right\} \mathrm{NMR}\left(100 \mathrm{MHz}, \mathrm{CDCl}_{3}\right) \square 19.7,51.2,64.9,67.1,123.8$ (q, $\left.J=3.8 \mathrm{~Hz}\right), 124.2(\mathrm{q}, J$ = 3.7 Hz), $124.2(\mathrm{q}, J=272.1 \mathrm{~Hz}), 128.8,130.6(\mathrm{q}, J=32.1 \mathrm{~Hz}), 130.9$, 145.3; Anal. Calcd for $\mathrm{C}_{13} \mathrm{H}_{16} \mathrm{~F}_{3} \mathrm{NO}$ : C, 60.22; H, 6.22; N, 5.40. Found: C, 59.95; H, 6.34; N, 5.66.

$N$-[1-(4-Trifluoromethylphenyl)ethyl]morpholine (Table 2, Entry 6). The general procedure was followed with 4-trifluoromethylstyrene (591 $\square 1,4.00 \mathrm{mmol})$, morpholine (87.1 $\mathrm{mg}, 1.00 \mathrm{mmol}$ ) and $0.20 \mathrm{~mL}$ of 1,4-dioxane. The reaction mixture was purified by flash column chromatography (20\% EtOAc in hexane) to give $126 \mathrm{mg}(48 \%)$ of the hydroamination product. ${ }^{1} \mathrm{H}$ NMR (400 MHz, $\mathrm{CDCl}_{3}$, TMS) $\square 1.34$ (d, $\left.J=6.8 \mathrm{~Hz}, 3 \mathrm{H}\right), 2.27-2.39$ (m, 2H), 2.42-2.57 (m, 
2H), 3.36 (q, $J=6.8 \mathrm{~Hz}, 1 \mathrm{H}), 3.62-3.77(\mathrm{~m}, 4 \mathrm{H}), 7.45$ (d, $J=8.1 \mathrm{~Hz}, 2 \mathrm{H}), 7.57(\mathrm{~d}, J=8.1 \mathrm{~Hz}$, $2 \mathrm{H}) ;{ }^{13} \mathrm{C}\left\{{ }^{1} \mathrm{H}\right\}$ NMR $\left(100 \mathrm{MHz}, \mathrm{CDCl}_{3}\right) \square 19.8,51.2,65.0,67.1,124.2$ (q, $\left.J=272.6 \mathrm{~Hz}\right), 125.3$ $(\mathrm{q}, J=4.0 \mathrm{~Hz}), 127.8,129.2(\mathrm{q}, J=31.8 \mathrm{~Hz}), 148.4$; Anal. Calcd for $\mathrm{C}_{13} \mathrm{H}_{16} \mathrm{~F}_{3} \mathrm{NO}: \mathrm{C}, 60.22 ; \mathrm{H}$, 6.22; N, 5.40. Found: C, 60.38; H, 6.04; N, 5.11.

$N$-(1-Phenylethyl)-4-phenylpiperazine (Table 2, Entry 7). The general procedure was followed with styrene $(229 \square 1,2.00 \mathrm{mmol})$ and 4-phenylpiperazine (163 mg, $1.00 \mathrm{mmol})$. The reaction mixture was purified by flash column chromatography (5\% EtOAc in hexane) to give $192 \mathrm{mg}(72 \%)$ of the hydroamination product as an oil. ${ }^{1} \mathrm{H}$ NMR (400 $\left.\mathrm{MHz}, \mathrm{CDCl}_{3}, \mathrm{TMS}\right)$ $1.39(\mathrm{~d}, J=6.8 \mathrm{~Hz}, 3 \mathrm{H}), 2.47-2.57(\mathrm{~m}, 2 \mathrm{H}), 2.58-2.68(\mathrm{~m}, 2 \mathrm{H}), 3.15(\mathrm{t}, J=5.0 \mathrm{~Hz}, 4 \mathrm{H}), 3.38(\mathrm{q}$, $J=6.8 \mathrm{~Hz}, 1 \mathrm{H}), 6.78-6.86(\mathrm{~m}, 1 \mathrm{H}), 6.87-6.93(\mathrm{~m}, 2 \mathrm{H}), 7.17-7.28(\mathrm{~m}, 3 \mathrm{H}), 7.27-7.37$ (m, 4H); ${ }^{13} \mathrm{C}\left\{{ }^{1} \mathrm{H}\right\}$ NMR $\left(100 \mathrm{MHz}, \mathrm{CDCl}_{3}\right) \square 20.0,49.2,50.6,64.9,115.8,119.5,126.9,127.6,128.2$, 129.0, 143.9, 151.3; Anal. Calcd for $\mathrm{C}_{18} \mathrm{H}_{22} \mathrm{~N}_{2}$ : C, 81.16; H, 8.32; N, 10.52. Found: C, 80.93; H, $8.41 ; \mathrm{N}, 10.22$.

N-(1-Phenylethyl)-4-'butylcarboxypiperazine (Table 2, Entry 8). The general procedure was followed with styrene (229 $\square 1,2.00 \mathrm{mmol})$ and 4-tert-butyl carboxypiperazine (189 $\mathrm{mg}, 1.02 \mathrm{mmol}$ ). The reaction mixture was stirred at $110{ }^{\circ} \mathrm{C}$ for $24 \mathrm{~h}$. The reaction mixture was purified by flash column chromatography (10\% EtOAc in hexane) to give $187 \mathrm{mg}(63 \%)$ of the hydroamination product. ${ }^{1} \mathrm{H}$ NMR (400 MHz, $\mathrm{CDCl}_{3}$, TMS) $\square 1.36(\mathrm{~d}, J=6.6 \mathrm{~Hz}, 3 \mathrm{H}), 1.43$ (s, 9H), 2.24-2.38 (m, 2H), 2.34-2.48 (m, 2H), 3.37 (q, J = 6.6 Hz, 1H), 3.37-3.43 (m, 4H), 7.20$7.28(\mathrm{~m}, 1 \mathrm{H}), 7.27-7.33(\mathrm{~m}, 4 \mathrm{H}) ;{ }^{13} \mathrm{C}\left\{{ }^{1} \mathrm{H}\right\} \mathrm{NMR}\left(100 \mathrm{MHz}, \mathrm{CDCl}_{3}\right.$ ) 1 19.6. 28.3, 43.6 (br), 50.2, 64.6, 79.3, 126.9, 127.5, 128.2, 143.5. 154.6.

N-1-(Phenylethyl)isoindoline (Table 2, Entry 9). ${ }^{4}$ The general procedure was followed with styrene (458 $\square 1,4.00 \mathrm{mmol})$ and isoindoline $(119 \mathrm{mg}, 1.00 \mathrm{mmol})$. The reaction mixture was purified by flash column chromatography (2\% EtOAc in hexane) to give $158 \mathrm{mg}(71 \%)$ of the hydroamination product. ${ }^{1} \mathrm{H}$ NMR (400 $\left.\mathrm{MHz} \mathrm{CDCl}_{3}, \mathrm{TMS}\right) \square 1.49$ (d, J = 6.5 Hz, 3H), 3.63 $(\mathrm{q}, \mathrm{J}=6.5 \mathrm{~Hz}, 1 \mathrm{H}), 3.75-3.84(\mathrm{~m}, 2 \mathrm{H}), 3.89-3.98(\mathrm{~m}, 2 \mathrm{H}), 7.16(\mathrm{~s}, 4 \mathrm{H}), 7.24-7.31(\mathrm{~m}, 1 \mathrm{H})$, 7.32$7.38(\mathrm{~m}, 2 \mathrm{H}), 7.28-7.44(\mathrm{~m}, 2 \mathrm{H}) ;{ }^{13} \mathrm{C}\left\{{ }^{1} \mathrm{H}\right\} \mathrm{NMR}\left(100 \mathrm{MHz}, \mathrm{CDCl}_{3}\right) \square$ 23.3, 58.0, 65.4, 122.3, 126.6, 127.1, 127.2, 128.5, 140.1, 145.2.

N-1-(Phenylethyl)tetrahydroisoquinoline (Table 2, Entry 10). The general procedure was followed with styrene $(229 \square 1,2.00 \mathrm{mmol})$ and 1,2,3,4-tetrahydroisoquinoline (136 mg, $1.02 \mathrm{mmol})$. The reaction mixture was purified by flash column chromatography $(2 \%$ EtOAc in 
hexane) to give $139 \mathrm{mg}(58 \%)$ of the hydroamination product. ${ }^{1} \mathrm{H} \mathrm{NMR}\left(400 \mathrm{MHz}, \mathrm{CDCl}_{3}\right.$, TMS) $\square 1.47$ (d, J = 6.8 Hz, 3H), 2.54-2.66 (m, 1H), 2.71-2.94 (m, 3H), 3.55 (q, J = 6.8 Hz, 1H), $3.56(\mathrm{~d}, \mathrm{~J}=14.6 \mathrm{~Hz}, 1 \mathrm{H}), 3.82$ (d, J = 15.1 Hz, 1H), 6.95-7.02 (m, 1H), 7.03-7.14 (m, 3H), 7.22$7.28(\mathrm{~m}, 1 \mathrm{H}), 7.29-7.36(\mathrm{~m}, 2 \mathrm{H}), 7.35-7.41(\mathrm{~m}, 2 \mathrm{H}) ;{ }^{13} \mathrm{C}\left\{{ }^{1} \mathrm{H}\right\}$ NMR $\left(100 \mathrm{MHz}, \mathrm{CDCl}_{3}\right) \square 20.2$, 29.3, 48.0, 53.6, 64.4, 125.5, 126.0, 126.8, 126.9, 127.5, 128.3, 128.6, 134.6, 135.1, 144.2; Anal. Calcd for $\mathrm{C}_{17} \mathrm{H}_{19} \mathrm{~N}$ : C, 86.03; H, 8.07; N, 5.90. Found: C, 85.81; H, 8.13; N, 5.79.

$N$-[1-(2-Naphthyl)ethyl]piperidine (Table 2, Entry 11). The general procedure was followed with vinylnaphthalene $(308 \mathrm{mg}, 2.00 \mathrm{mmol})$ and piperidine $(84.5 \mathrm{mg}, 0.992 \mathrm{mmol})$. The reaction mixture was stirred at $110{ }^{\circ} \mathrm{C}$ for $24 \mathrm{~h}$. The reaction mixture was purified by flash column chromatography (10\% EtOAc in hexane) to give $124 \mathrm{mg}(52 \%)$ of the hydroamination product. ${ }^{1} \mathrm{H}$ NMR (400 MHz, $\mathrm{CDCl}_{3}$, TMS) $\square$ 1.33-1.42 (m 2H), 1.43 (d, J = 6.6 Hz, 3H), 1.491.59 (m, 4H), 2.26-2.43 (m, 2H), 2.36-2.52 (m, 2H), 3.52 (q, J = 6.6 Hz, 1H), 7.28-7.48 (m, 2H), $7.50(\mathrm{~d}, \mathrm{~J}=8.4 \mathrm{~Hz}, 1 \mathrm{H}), 7.69(\mathrm{~s}, 1 \mathrm{H}), 7.79(\mathrm{t}, \mathrm{J}=7.9 \mathrm{~Hz}, 3 \mathrm{H}) ;{ }^{13} \mathrm{C}\left\{{ }^{1} \mathrm{H}\right\} \mathrm{NMR}\left(100 \mathrm{MHz}, \mathrm{CDCl}_{3}\right)$ $\square$ 19.4, 24.6, 26.2, 51.7, 65.3, 125.3, 125.7, 126.1, 126.2, 127.5, 127.6, 127.7, 132.6, 133.2, 141.8; Anal. Calcd for $\mathrm{C}_{17} \mathrm{H}_{21} \mathrm{~N}$ : C, 85.30; H, 8.84; N, 5.85. Found: C, 85.62; H, 8.65; N, 5.64.

$N$-[1-(2-naphthyl)ethyl]benzylmethylamine (Table 2, Entry 12). The general procedure was followed with 2-vinylnaphtalene $(308 \mathrm{mg}, 2.00 \mathrm{mmol})$ and $N$-benzyl methylamine $(122 \mathrm{mg}, 1.00 \mathrm{mmol})$. The reaction mixture was stirred at $80{ }^{\circ} \mathrm{C}$ for $24 \mathrm{~h}$. The reaction mixture was purified by flash column chromatography (4\% EtOAc in hexane) to give $175 \mathrm{mg}(63 \%)$ of the hydroamination product. ${ }^{1} \mathrm{H}$ NMR (400 $\left.\mathrm{MHz} \mathrm{CDCl}_{3}, \mathrm{TMS}\right) \square 1.49$ (d, J = 6.7 Hz, 3H), 2.16 (s, 3H), 3.34 (d, J = 13.6 Hz, 1H), 3.59 (d, J = 13.6 Hz, 1H), 3.77 (q, J = 6.7 Hz, 1H), 7.15-7.23 $(\mathrm{m}, 1 \mathrm{H}), 7.24-7.34(\mathrm{~m}, 4 \mathrm{H}), 7.37-7.46(\mathrm{~m}, 2 \mathrm{H}), 7.58-7.66(\mathrm{~m}, 1 \mathrm{H}), 7.74-7.84(\mathrm{~m}, 4 \mathrm{H}) ;{ }^{13} \mathrm{C}\left\{{ }^{1} \mathrm{H}\right\}$ NMR (100 MHz, $\left.\mathrm{CDCl}_{3}\right) \square 18.2,38.4,58.9,63.3,125.4,125.8,125.9,126.2,126.7,127.6$, 127.8, 127.8, 128.1, 128.7, 132.7, 133.3, 139.9, 142.0; Anal. Calcd for $\mathrm{C}_{20} \mathrm{H}_{21} \mathrm{~N}$ : C, 87.23; H, 7.69; N, 5.09. Found: C, 87.08; H, 7.92; N, 5.04.

$N$-[1-(4-Methylphenyl)ethyl]benzylmethylamine (Table 2, Entry 13). The general procedure was followed with 4-methylstyrene (527 $\square 1,4.00 \mathrm{mmol}), N$-methyl benzylamine (121 $\mathrm{mg}, 1.00 \mathrm{mmol}$ ) and $0.20 \mathrm{~mL}$ of 1,4-dioxane. The reaction mixture was stirred at $110{ }^{\circ} \mathrm{C}$ for 24 h. The reaction mixture was purified by flash column chromatography (10\% EtOAc in hexane) to give $129 \mathrm{mg}$ of a mixture of the hydroamination product and dibenzylmethylamine consisting of a 4.3:1 ratio of the hydroamination product to dibenzylmethylamine, as determined by ${ }^{1} \mathrm{H}$ 
NMR spectroscopy. These quantities corresponded to a $43 \%$ yield of the hydroamination product. $N$-[1-(4-methylphenyl)ethyl]benzylmethylamine was difficult to separate from dibenzylmethylamine in high yield, but $43.1 \mathrm{mg}(18 \%)$ of a pure sample of $N$-[1-(4methylphenyl)ethyl]benzylmethylamine was isolated from the reaction mixture by selectively combining fractions collected by flash column chromatography (2\% EtOAc in hexane). ${ }^{1} \mathrm{H} \mathrm{NMR}$ $\left(400 \mathrm{MHz}, \mathrm{CDCl}_{3}, \mathrm{TMS}\right) \square 1.41(\mathrm{~d}, J=6.9 \mathrm{~Hz}, 3 \mathrm{H}), 2.12(\mathrm{~s}, 3 \mathrm{H}), 2.34(\mathrm{~s}, 3 \mathrm{H}), 3.27$ (d, $J=12.9$ $\mathrm{Hz}, 1 \mathrm{H}), 3.58$ (d, $J=13.1 \mathrm{~Hz}, 1 \mathrm{H}), 3.61$ (q, $J=6.8 \mathrm{~Hz}, 1 \mathrm{H}), 7.15$ (d, $J=7.8 \mathrm{~Hz}, 2 \mathrm{H}), 7.18-7.36$ $(\mathrm{m}, 7 \mathrm{H}) ;{ }^{13} \mathrm{C}\left\{{ }^{1} \mathrm{H}\right\}$ NMR $\left(100 \mathrm{MHz}, \mathrm{CDCl}_{3}\right) \square 18.5,21.1,38.3,58.7,62.9,126.7,127.6,128.1$, 128.2, 128.7, 128.8, 128.9, 136.3; Anal. Calcd for $\mathrm{C}_{17} \mathrm{H}_{21} \mathrm{~N}$ : C, 85.30; H, 8.84; N, 5.85. Found: C, 85.39; H, 8.80; N, 5.57.

N-[1-(2-naphthyl)ethyl]hexylmethylamine (Table 2, Entry 14). The general procedure was followed with 2-vinylnaphtalene $(308 \mathrm{mg}, 2.00 \mathrm{mmol})$ and $N$-hexyl methylamine $(116 \mathrm{mg}$, $1.01 \mathrm{mmol})$. The reaction mixture was stirred at $110{ }^{\circ} \mathrm{C}$ for $18 \mathrm{~h}$ and was then purified by flash column chromatography (2\% EtOAc in hexane) to give $144 \mathrm{mg}(53 \%)$ of the hydroamination product. ${ }^{1} \mathrm{H} \mathrm{NMR}\left(400 \mathrm{MHz}, \mathrm{CDCl}_{3}\right.$, TMS) $\square 0.84$ (t, J = 6.8 Hz, 3H), 1.17-1.27 (m, 6H), 1.39$1.52(\mathrm{~m}, 2 \mathrm{H}), 1.42(\mathrm{~d}, \mathrm{~J}=6.7 \mathrm{~Hz}, 3 \mathrm{H}), 2.22(\mathrm{~s}, 3 \mathrm{H}), 2.27-2.34(\mathrm{~m}, 1 \mathrm{H}), 2.37-2.48(\mathrm{~m}, 1 \mathrm{H}), 3.68$ $(\mathrm{q}, \mathrm{J}=6.5 \mathrm{~Hz}, 1 \mathrm{H}), 7.37-7.48(\mathrm{~m}, 2 \mathrm{H}), 7.49-7.55(\mathrm{~m}, 1 \mathrm{H}), 7.70(\mathrm{~s}, 1 \mathrm{H}), 7.74-7.84(\mathrm{~m}, 3 \mathrm{H})$; ${ }^{13} \mathrm{C}\left\{{ }^{1} \mathrm{H}\right\} \operatorname{NMR}\left(100 \mathrm{MHz}, \mathrm{CDCl}_{3}\right) \square 14.1,18.6,22.6,27.1,27.1,31.8,38.7,54.5,63.5,125.3$, 125.7, 125.9, 126.3, 127.5, 127.7, 127.7, 132.6, 133.3, 142.1; Anal. Calcd for $\mathrm{C}_{19} \mathrm{H}_{27} \mathrm{~N}$ : C, 84.70; H, 10.10; N, 5.20. Found: C, 84.58; H, 9.96; N, 5.33.

Synthesis of $\{[D P P F] P d[1-(4-m e t h y l p h e n y l) e t h y l]\}$ triflate (1). In a dry box, $\mathrm{Pd}(\mathrm{DPPF})(\mathrm{OTf})_{2}(143 \mathrm{mg}, 0.15 \mathrm{mmol})$ was suspended in $3.0 \mathrm{~mL}$ of toluene in a screw-capped vial. 4-Methylstyrene (295 $\square 1,2.24 \mathrm{mmol})$ and 4-trifluoromethylaniline (188 $\square 1,1.50 \mathrm{mmol})$ were added to the suspension. The vial was sealed with a cap containing a PTFE septum and removed from the dry box. The suspension was stirred at $80{ }^{\circ} \mathrm{C}$ for $12 \mathrm{~h}$. After heating, the reaction mixture was added to $20 \mathrm{~mL}$ of pentane while stirring in a dry box, and an orange precipitate was obtained by filtration. The crude product was washed repeatedly with pentane and diethyl ether, and was recrystallized from toluene at $-30{ }^{\circ} \mathrm{C}$. Complex $\mathbf{1}$ was obtained as an orange solid (29.1 mg, $0.031 \mathrm{mmol}$ ) in $21 \%$ yield. ${ }^{1} \mathrm{H}$ NMR (400 MHz, acetone- $d_{6}$, TMS) $\square 0.90$ (ddd, $J=12.6,11.3,6.8 \mathrm{~Hz}, 3 \mathrm{H}), 1.87$ (dd, $J=4.4,2.3 \mathrm{~Hz}, 3 \mathrm{H}), 3.67-3.72(\mathrm{~m}, 1 \mathrm{H}), 3.83-3.88$ $(\mathrm{m}, 1 \mathrm{H}), 4.85(\mathrm{br} \mathrm{q}, J=11.3 \mathrm{~Hz}, 1 \mathrm{H}), 4.35-4.42(\mathrm{~m}, 2 \mathrm{H}), 4.63-4.67(\mathrm{~m}, 1 \mathrm{H}), 4.67-4.72(\mathrm{~m}, 1 \mathrm{H})$, 
4.73-4.78 (m, 1H), 4.83-4.87 (m, 1H), $6.80(\mathrm{~d}, J=7.8 \mathrm{~Hz}, 1 \mathrm{H}), 7.06-7.21(\mathrm{~m}, 5 \mathrm{H})$, 7.36-7.51 (m, 4H), 7.51-7.75 (m, 14H); ${ }^{31} \mathrm{P}$ NMR (121.6 MHz, d $\mathrm{d}^{6}$-acetone) $\square 32.4(\mathrm{~d}, J=51.3 \mathrm{~Hz}), 21.9(\mathrm{~d}, J=$ $51.3 \mathrm{~Hz}$ ); Anal. Calcd for $\mathrm{C}_{44} \mathrm{H}_{39} \mathrm{~F}_{3} \mathrm{FeO}_{3} \mathrm{P}_{2} \mathrm{PdS}$ : C, 56.88; H, 4.23. Found: C, 57.13; H, 4.21.

$N$-[1-(4-Methylphenyl)ethyl]morpholine (equation 2). An authentic sample of the organic product from eq. 2 was synthesised according to the general procedure used to prepare the products in Table 2. The general procedure was followed with 4-methylstyrene (264 $\square 1,2.00$ mmol) and morpholine $(87.3 \mathrm{mg}, 1.00 \mathrm{mmol})$. The reaction mixture was stirred at $110{ }^{\circ} \mathrm{C}$ for 24 h. The reaction mixture was purified by flash column chromatography (20\% EtOAc in hexane) to give $159 \mathrm{mg}(77 \%)$ of the hydroamination product. ${ }^{1} \mathrm{H}$ NMR (400 $\left.\mathrm{MHz}, \mathrm{CDCl}_{3}, \mathrm{TMS}\right) \square 1.34$ $(\mathrm{d}, J=6.8 \mathrm{~Hz}, 3 \mathrm{H}), 2.33(\mathrm{~s}, 3 \mathrm{H}), 2.35(\mathrm{~m}, 2 \mathrm{H}), 2.47(\mathrm{~m}, 2 \mathrm{H}), 3.26(\mathrm{q}, J=6.8 \mathrm{~Hz}, 1 \mathrm{H}), 3.68(\mathrm{t}, J$ $=4.7 \mathrm{~Hz}, 4 \mathrm{H}), 7.20(\mathrm{~d}, J=8.1 \mathrm{~Hz}, 2 \mathrm{H}), 7.19(\mathrm{~d}, J=8.1 \mathrm{~Hz}, 2 \mathrm{H}) ;{ }^{13} \mathrm{C}\left\{{ }^{1} \mathrm{H}\right\} \mathrm{NMR}(100 \mathrm{MHz}$, $\left.\mathrm{CDCl}_{3}\right) \square 19.8,21.0,51.2,65.0,67.2$, 127.5, 128.9, 136.5, 140.7; Anal. Calcd for $\mathrm{C}_{13} \mathrm{H}_{19} \mathrm{NO}$ : C, 76.06; H, 9.33; N, 6.82. Found: C, 75.85; H, 9.56; N, 7.03.

Reaction of complex 1 with morpholine in the presence of styrene. In a dry box,

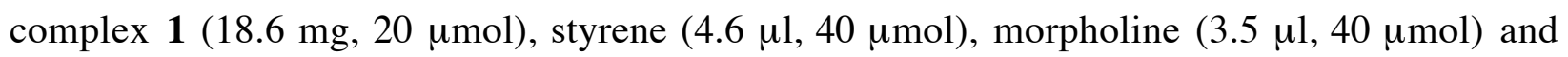
dodecane $(7.1 \mathrm{mg}, 42 \square \mathrm{mol})$ as internal standard were suspended in $0.20 \mathrm{~mL}$ of dioxane in a screw-capped vial. The vial was sealed with a cap containing a PTFE septum and removed from the dry box. The reaction mixture was stirred at $110{ }^{\circ} \mathrm{C}$, and the formation of product was monitored by GC analysis. The data are summarised in Table 1.

Table 1. Product distribution from the reaction of complex $\mathbf{1}$ with morpholine in the presence of styrene.

\begin{tabular}{|c|c|c|}
\hline Time / $\min$ & $\begin{array}{c}\text { N-[1-(4-methylphenyl)ethyl]morpholine } \\
\times 10^{2} \mathrm{M}\end{array}$ & $\begin{array}{c}\text { N-1-(phenethyl)morpholine } \\
\times 10^{2} \mathrm{M}\end{array}$ \\
\hline 1 & 1.1 & 0.0 \\
5 & 1.1 & 0.4 \\
10 & 1.5 & 0.9 \\
15 & 1.6 & 1.2 \\
20 & 1.6 & 1.4 \\
\hline
\end{tabular}

Enantioselective hydroaminaton (eq. 3). In a dry box, $\mathrm{Pd}\left(\mathrm{O}_{2} \mathrm{CCF}_{3}\right)_{2}(35.4 \mathrm{mg}, 0.080$ $\mathrm{mmol})$ and R, R-Et-FerroTANE (35.4 mg, $0.080 \mathrm{mmol}$ ) were suspended in $0.10 \mathrm{~mL}$ of dioxane in a screw-capped vial. 2-Vinylnaphthalene (493 $\mathrm{mg}, 3.20 \mathrm{mmol})$ and $N$-benzylmethylamine $(97.8 \mathrm{mg}, 0.81 \mathrm{mmol})$ were added to into another small vial and dissolved in $0.20 \mathrm{~mL}$ of $1,4-$ dioxane. The vials were sealed with a cap containing a PTFE septum and removed from the dry 
box. Triflic acid $(35.5 \square 1,0.40 \mathrm{mmol})$ was added to the catalyst suspension, followed by the solution of vinylarenes and amines. The reaction mixture was stirred at $50{ }^{\circ} \mathrm{C}$ for $48 \mathrm{~h}$. After heating, the reaction mixture was purified by flash column chromatography (2\% EtOAc in hexane) to give $80.0 \mathrm{mg}(36 \%)$ of the hydroamination product. The enantiomeric excess was determined to be $63 \%$ by HPLC analysis with a Daicel CHIRALCEL OJ column (hexanes/ipropanol $=99.5 / 0.5)$.

(1) Stang, P. J.; Olenyuk, B.; Arif, A. M. Organometallics 1995, 14, 5281.

(2) Alcazar-Roman, L. M.; Hartwig, J. F.; Rheingold, A. L.; Liable-Sands, L. M.; Guzei, I. A. J. Am. Chem. Soc. 2000, 122, 4618.

(3) Kawaguchi, M.; Ohashi, J.; Kawakami, Y.; Oda, J. Synthesis 1985, 701.

(4) Suwa, T.; Sugiyama, E.; Baba, A. Synthesis 2000, 789. 